\title{
Multiparametric MRI in the management of prostate cancer: an update-a narrative review
}

\author{
Francesco Ziglioli ${ }^{1}$, Umberto Maestroni ${ }^{1}$, Carmelinda Manna ${ }^{2}$, Giulio Negrini ${ }^{2}$, Giorgia Granelli ${ }^{1}$, \\ Valentina Greco ${ }^{2}$, Francesco Pagnini ${ }^{2}$, Massimo De Filippo ${ }^{2}$ \\ ${ }^{1}$ Department of Urology, University-Hospital of Parma, Parma, Italy; ${ }^{2}$ Department of Radiology, University-Hospital of Parma, Parma, Italy \\ Contributions: (I) Conception and design: F Ziglioli, U Maestroni, M De Filippo, C Manna; (II) Administrative support: None; (III) Provision of \\ study materials or patients: V Greco, G Granelli, C Manna; (IV) Collection and assembly of data: V Greco, G Granelli, G Negrini; (V) Data analysis \\ and interpretation: U Maestroni, M De Filippo, U Maestroni, G Negrini; (VI) Manuscript writing: All authors; (VII) Final approval of manuscript: \\ All authors. \\ Correspondence to: Massimo De Filippo. Department of Radiology, University-Hospital of Parma, Via Gramsci, 14, 43126 Parma, Italy. \\ Email: massimo.defilippo@unipr.it.
}

\begin{abstract}
The growing interest in multiparametric MRI is leading to important changes in the diagnostic process of prostate cancer. MRI-targeted biopsy is likely to become a standard for the diagnosis of prostate cancer in the next years. Despite it is well known that MRI has no role as a staging technique, it is clear that multiparametric MRI may be of help in active surveillance protocols. Noteworthy, MRI in active surveillance is not recommended, but a proper understanding of its potential may be of help in achieving the goals of a delayed treatment strategy. Moreover, the development of minimally invasive techniques, like laparoscopic and robotic surgery, has led to greater expectations as regard to the functional outcomes of radical prostatectomy. Multiparametric MRI may play a role in planning surgical strategies, with the aim to provide the highest oncologic outcome with a minimal impact on the quality of life. We maintain that a proper anatomic knowledge of prostate lesions may allow the surgeon to achieve a better result in planning as well as in performing surgery and help the surgeon and the patient engage in a shared decision in planning a more effective strategy for prostate cancer control and treatment. This review highlights the advantages and the limitations of multiparametric MRI in prostate cancer diagnosis, in active surveillance and in planning surgery.
\end{abstract}

Keywords: Multiparametric MRI; tageted biopsy; prostate cancer; extraprostatic extension; active surveillance

Submitted Jun 14, 2020. Accepted for publication Nov 11, 2020.

doi: $10.21037 / g s-20-561$

View this article at: http://dx.doi.org/10.21037/gs-20-561

\section{Introduction}

The advent of PSA testing more than two decades ago has improved early detection of prostate cancer, leading to more men being diagnosed and treated (1-15).

Interestingly, it is still controversial whether the increased detection and treatment of prostate cancer has led to increased overall survival rates. Data from two longterm screening studies were published in the last few years and reported conflicting results. The Prostate, Lung, Colorectal and Ovarian screening concluded that there is no difference between men who were screened and men who were not screened (16). On the other hand, the European Randomized Study of Screening for Prostate Cancer found a $20 \%$ reduction in the mortality rate in screened men (17).

For years, Trans-Rectal Ultrasound Guided biopsy (TRUS-Gb) has been the gold standard for the detection of prostate cancer in men with increased level of PSA or positive Digital Rectal Examination (DRE). The development of more and more sophisticated methods for the diagnosis and treatment (18-29) of prostate cancer, 


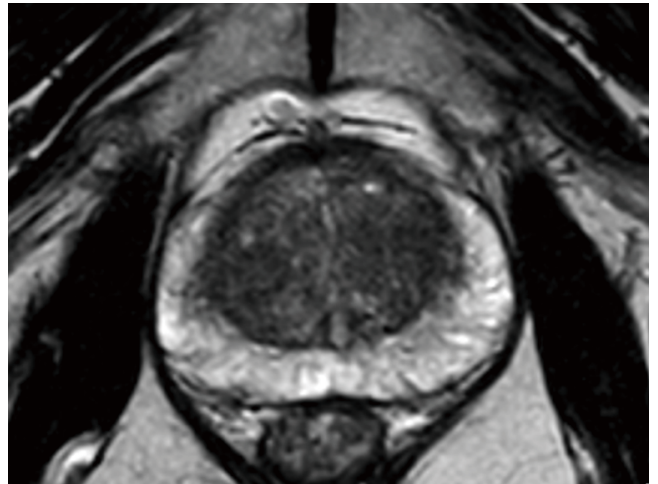

Figure 1 Axial T2-weighted image (T2WI) showing the normal hyperintense T2 signal in peripheral zone and low or disomogeneous signal of transitional zone. No lesion is present.

including imaging, led to focusing more on the detection rather than on the staging of this disease. At the same time, the advent of the Multiparametric MRI allowed to do a step forward in the detection process. The shift from staging to detection, along with the shift from TRUS-Gb to MRI Targeted-biopsy (MRI-Tb) represents the so called "paradigm shift".

Due to its diagnostic performance, which is higher in high-risk prostate cancer, multiparametric MRI and targeted biopsy (30-33) may play a role in reducing overdiagnosis and overtreatment on a large scale.

The aim of this narrative review is to give an update on the diagnostic pathways of prostate cancer detection and provide a picture of the cutting-edge imaging methods and their application to clinical practice.

\section{Methods}

Four electronic databases (PubMed, Medline, Embase via Ovid and Cochrane review database) were searched from January 2000 to April 2020. The search strategies were: multiparametric MRI; fusion biopsy + prostate cancer; targeted biopsy + prostate cancer; extraprostatic extension, extracapsular extension + MRI; active surveillance + MRI.

The preferred citations were meta-analysis and systematic reviews on the given topics. Only articles in English language were considered.

\section{Results}

Our search yielded 63 reports, about which we comment, below.

\section{Multiparametric MRI performance in detecting Prostate Cancer}

In 2014, the International Society of Urological Pathology (ISUP) modified the grading system for prostate cancer (34), in order to further define the clinically highly significant groups of prostate cancer, and subdivided prostate cancer in five different groups on the basis of Gleason score grading system (35).

Even if there is not common agreement in the literature about the definition of "clinically significant" prostate cancer, it is generally accepted by the majority of the Authors that Gleason score $\geq 3+3$ (ISUP $\geq 2$ ) may be considered "significant".

A systematic review carried out by Valerio et al. in 2015 showed that MRI-targeted biopsy (MRI-Tb) detects a higher number of clinically significant prostate cancers with a lower number of cores compared with TRUS-Gb. The review included 14 papers with a total of 2,293 patients. MRI-Tb detected $6.8 \%$ more clinically significant prostate cancer than TRUS-Gb (36). In addition, the median number of specimens necessary to detect one prostate cancer was 37.1 for TRUS-Gb and 9.2 for MRI-Tb, thus showing that systematic mapping of the prostate would require approximately four times the number of cores compared to MRI-Tb to diagnose one prostate cancer.

\section{Multiparametric MRI performance in detecting ISUP Grade 1 Prostate Cancer versus ISUP Grade $\geq 2$ Prostate Cancer}

The performance of multiparametric MRI correlates with the ISUP risk classification of prostate cancer.

In fact, multiparametric MRI is more sensitive in detecting ISUP Grade $\geq 2$ than ISUP Grade 1 prostate cancer (Figures 1-4). This is particularly evident for small size prostate cancers. If we consider the subgroup of cancers smaller than $0.5 \mathrm{~cm}$, multiparametric MRI is reported to identify less than $30 \%$ of ISUP Grade 1 cancers, according with the results reported by Bratan et al. on histopathologic analysis of specimens retrieved by radical prostatectomy (37).

Compared to systematic biopsy, MRI-based targeted biopsy allowed to decrease the number of patients diagnosed with clinically-insignificant prostate cancer according with the results of the MRI-FIRST study (38). Similarly, the PRECISION and $4 M$ study found that the detection rate of ISUP Grade 1 prostate cancer was lower 


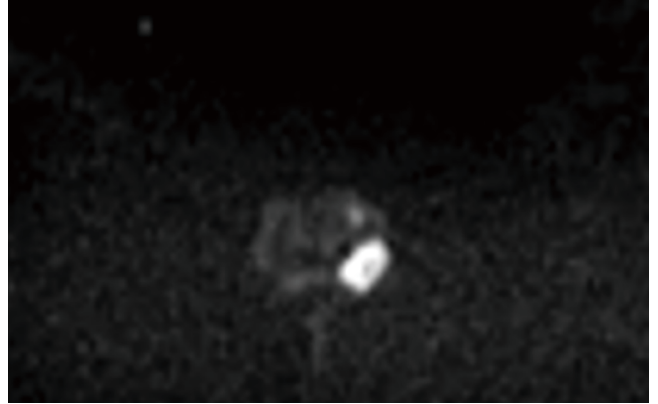

Figure 2 DW imaging of prostate cancer. Axial DWI b 2000 obtained at midprostate level showing a well evident focal high signal in $\mathrm{PZ}$ suggestive for malignancy.

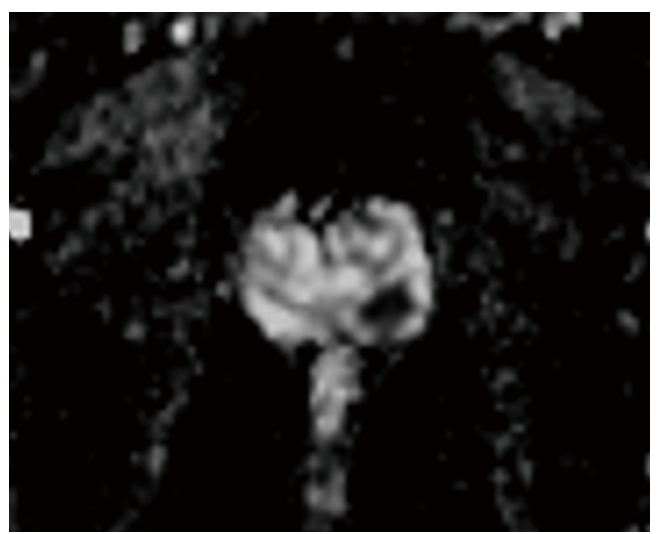

Figure 3 Diffusion-weighted imaging of prostate cancer. Axial ADC maps $\left(2400 / 81 ; \mathrm{b}=0,50,1,500 \mathrm{sec} / \mathrm{mm}^{2}\right)$ obtained at midprostate level in same patients.

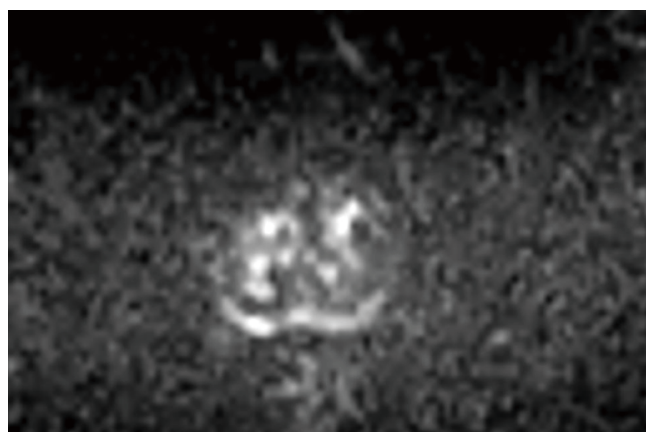

Figure 4 DW imaging of prostate cancer. Axial diffusion-weighted imaging b 2000 showing focal high signal in right PZ mid gland not easy to detect, suggestive for malignancy. in patients investigated with MRI-Tb than in the ones who underwent standard bioptic mapping. In details, the number of patients diagnosed with ISUP Grade 1 prostate cancer was $9 \%$ versus $22 \%$ and $14 \%$ versus $25 \%$, respectively (39).

If these data are analyzed in the frame of the much discussed overdiagnosis and overtreatment, it is clear that MRI targeted biopsy plays a role in reducing overdiagnosis of low-risk prostate cancer when compared to systematic biopsy $(40,41)$.

van der Leest et al. showed that in the setting of a template biopsy as a reference standard, multiparametric MRI has a sensitivity of 0.70 (95\% CI: 0.59-0.80) and a specificity of 0.27 (95\% CI: $0.19-0.37$ ) for the detection of ISUP Grade 1 prostate cancer (42).

\section{Correlation with radical prostatectomy specimens}

One of the most discussed topic in staging prostate cancer is Gleason score upgrading from biopsy to radical prostatectomy. It is well known that upgrading of Gleason score from preoperative biopsy (T1c) obtained by preoperative systematic bioptic mapping (10 to 12 sample cores) to postoperative histology after radical surgery is around $35 \%$, ranging from $14 \%$ to $51 \%$, according with Budäus et al. (43).

Many causes of Gleason score upgrading have been advocated. One of the most studied is the presence of the cribriform pattern, that is associated with a high number of cases of Gleason score upgrading. However, other factors have been documented, like patient age, prostate volume, and the number of bioptic cores, all influencing the Gleason score upgrading (44).

As a consequence, the decision-making process based on preoperative histological reports may be altered in favor of overtreatment or undertreatment of prostate cancer.

In this view, multiparametric MRI may be of help, as it has recently been shown to play a role in the detection and characterization of prostate cancer (45). We can maintain that when it is used on a routinary basis, multiparametric MRI is effective in detecting those tumors which are likely to result in an upgrading after surgical treatment. Radical prostatectomy data show that a positive multiparametric MRI is more likely to be associated with upgrading (Gleason score $\geq 6$ ) than a negative MRI (43\% versus $27 \%$ ) (46). 


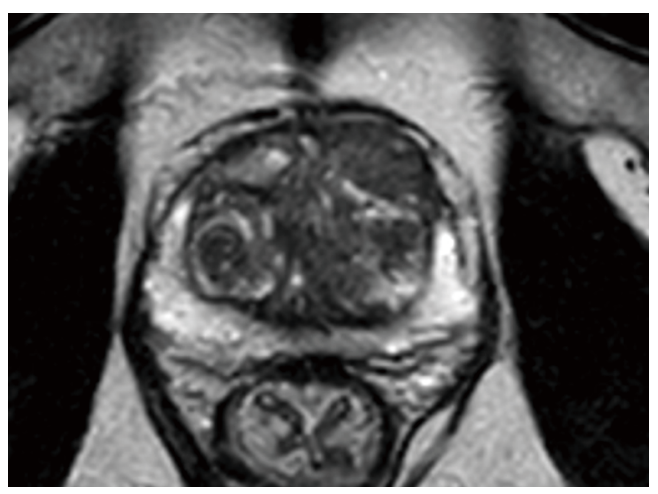

Figure 5 Extracapsular extension of prostate tumor. Axial T2weighted image (T2WI) showing the low signal tumor in the anterior zone with minimal bulging along the capsule

Song et al. in 2018 reported that PIRADS score v.2 has a high predictive value in predicting Gleason score upgrading. However, this study is retrospective and all patients underwent multiparametric MRI around 20 days after a prostate bioptic mapping was performed. It is well known that inflammation and local intra-prostatic haemorrage are a common cause of imaging misinterpretation (47).

A couple of years later, Alqahtani et al. carried out a study on 330 patients in order to evaluate the predictive accuracy of pre-bioptic multiparametric MRI in Gleason score upgrading after Radical Prostatectomy. This study showed that PIRADS v2 score has a great impact in predicting Gleason score update, followed by PSA level (48).

Since D'Amico pioneered this approach (49), the introduction of nomograms and algorithms in clinical practice in known to improve the ability of prognosticating the outcome of a treatment or the probability of recurrence and many are reported in the literature, also for experimental techniques (50).

Including the PIRADS score in preoperative nomograms is likely to allow clinicians to achieve great benefits in the decision-making process, according to what is reported in the literature so far.

\section{Multiparametric MRI and extra-prostatic tumor extension (EPE)}

Positive surgical margins are associated to biochemical recurrence and a reduction in overall survival (OS) after radical prostatectomy $(51,52)$.

Feng et al. compared the accuracy of multiparametric
MRI with Partin Tables and Memorial Sloan Kettering Cancer Center nomogram and concluded for a minor improvement in diagnostic accuracy after the addition of multiparametric MRI to the model. However, the patients enrolled into this study were mainly at low risk and thus with lower rates of extracapsular extension (53).

Gupta et al. compared the accuracy of MRI and Partin Tables in predicting EPE, but did not analyze the additional value of MRI to Partin Tables. The Authors compared these two tools as alternative models, concluding that Partin Tables have an accuracy of 0.62 (AUC) and MRI an accuracy of 0.82 (AUC) (54).

For these reasons, the role of multiparametric MRI in predicting EPE is not clearly defined, even if the interest in this technique is growing (55) (Figure 5).

Reportedly, however, the current guidelines issued by the European Association of Urology (56) and by the National Comprehensive Cancer Network (57) do not provide definitive indications in support of a routinary use of multiparametric MRI for the prediction of EPE at the time of radical prostatectomy and only hypothesize a role for this imaging technique, especially in the high risk cancer group.

Over the last years, some grading systems have been proposed in the aim of predicting extraprostatic tumor extension. The EPE grade demonstrated a good performance with a AUC of 0.77 (58). The ESUR score, also, showed a AUC around 0.77 in the most recent studies (59). The Likert scale is known to have a greater variability of performance, probably due to the lack of objective criteria (AUC 0.66-0.82) (58). The Capsular Contact Length (CCL) on multiparametric MRI has been proposed as a marker of EPE at pathology. A CCL $\geq 15 \mathrm{~mm}$ is considered to be significant $(60,61)$.

de Rooij et al. carried out a meta-analysis showing that multiparametric MRI had high specificity (91\%), but low sensitivity (only 57\%) in the detection of EPE. However, the studies included in this meta-analysis investigated prostate cancer with 1.5 Tesla MRI (62).

The lack of properly validated systems in EPE imaging interpretation is one of the main issue as regard to the performance of multiparametric MRI, as it is the main cause of the inter-reader variability. According with Park et al., among the grading systems for EPE, despite its simplicity, EPE grade is the system with the higher performance and correlates well with the histologic extent of $\operatorname{EPE}(63)$. 


\section{Multiparametric MRI when planning radical prostatectomy}

Traditionally, nerve-sparing radical prostatectomy is offered to patients with well differentiated and low risk cancer. The sparing techniques are known to reduce the negative impact of radical surgery on the quality of life.

Nonetheless, positive margins after radical surgery are widely reported to be a significant risk factor for prostate cancer recurrence. As a consequence, accurate prediction of EPE is useful when planning radical surgery and in estimating patient's prognosis.

For this reason, all the tools able to provide details on EPE before treatment may be of help in evaluating benefits and harms of nerve-sparing techniques and in tailoring treatment strategies in function of patient's characteristics (64).

Neuro-vascular bundles (NVB) are known to run posterolateral to the prostate from the base to the apex, except anatomical variation, that are hard to predict. In order to perform a "conservative" excision of the prostate, it is of the utmost importance for the surgeon to be sure of the absence of disease close to the posterolateral margins of the prostate (65).

In these respects, Schiavina et al. found that in approximately half the cases the nerve-sparing strategy may change if the multiparametric MRI imaging is taken into account at the time of planning the surgical procedure. In addition, in this study, it is reported that in $75 \%$ of the cases the strategy change was appropriate (66).

This study follows a previous series from Panebianco et al., where the Authors maintained that preoperative multiparametric MRI is able to support the surgeon in planning the appropriate surgical technique and may increase the quality of excision in up to $95.9 \%$ of the patients (67).

\section{Multiparametric MRI and active surveillance}

The role of multiparametric MRI before starting active surveillance

Active surveillance as well as watchful waiting are strategies to delay treatment or conservatively manage localized prostate cancer, respectively, in selected patients. Active monitoring has been proposed as an alternative option for differed treatment and differs from active surveillance in that PSA increases $\geq 50 \%$ in 12 months (68).
Around $45 \%$ of men diagnosed with prostate cancer are good candidates to active surveillance $(69,70)$, with the advantage to avoid immediate treatment if not necessary, without losing the opportunity to treat with a radical, curative intent.

The criteria for active surveillance for men diagnosed with localized prostate cancer and fit for radical surgery are: PSA $\leq 10 \mathrm{ng} / \mathrm{mL}$; PSA density $\leq 0.2 \mathrm{ng} / \mathrm{mL}^{2}$; Gleason score $\leq$ $6(3+3)$; clinical stage cT1c or cT2; less than 2 cores positive for adenocarcinoma (71).

Multiparametric MRI provides detailed information on the microvascular properties of the prostatic tissue (dynamic contrast-enhanced imaging, DCE) and facilitates lesion characterization (diffusion-weighted imaging, DWI).

Even if many Authors reported the correlation between old age and high PSA density, with a cut-off of $0.08 \mathrm{mg} / \mathrm{mL}^{2}$ according with the San Francisco validated threshold value (72), and the outcome of Active surveillance, there is no robust data about the impact of visible/non visible tumor at the multiparametric MRI.

In this respect, Park et al. reported that tumor visibility on multiparametric MRI was a significant independent predictor of unfavorable disease, thus suggesting that patients with prostate cancer without a discrete tumor on multiparametric MRI are more suitable for enrollment in an active surveillance protocol than those with a visible tumor $(\mathrm{P}<0.001)$ (73). Interestingly, Park et al. concluded that multiparametric MRI, together with other criteria, could be used to identify patients with prostate cancer eligible for active surveillance as an initial management strategy.

\section{The role of multiparametric MRI during active surveillance}

If the decision-making process from diagnosis to treatment were based on PSA testing only, this would lead to more men being overtreated; on the other hand, men with favorable (potentially insignificant) prostate cancer may harbor high risk grade cancer cells and miss their opportunity to receive radical treatment.

Traditionally, the active surveillance protocol is based on PSA kinetic, DRE and repeat biopsy.

However, the last edition of the European Association of Urology (EAU) Guidelines recommends the multiparametric MRI in the protocol for active surveillance in men with low-risk prostate cancer and attributes to this imaging technique a role in the management of delayed 
treatment and in deciding the appropriate timing for biopsy (56).

Noteworthy, the addition of multiparametric MRI imaging to the workup for active surveillance may reduce overtreatment and help protect patients from cancer progression.

The results from the ASIST study (74) show 50\% reduction of active surveillance failure in MRI cohort compared to standard biopsy cohort in highly experienced centers over a 2 years follow-up. Interestingly, the same study highlights a reduction in the Gleason upgrading rate in the MRI cohort. As a consequence, the usage of multiparametric MRI and MRI targeted biopsy can be considered a safer strategy to manage patient on active surveillance.

One of the main concerns of active surveillance is the timing for repeating biopsy. The usage of MRI targeted biopsy carries the advantage of decreasing the number repeat biopsies as much as $68 \%$, Siddiqui et al. report (75).

Shoots et al. confirm the results of the study carried out by Siddiqui et al., also finding a 10\% increase in the Gleason upgrading, that conflicts with the results of the ASIST study (76).

\section{Conclusions}

The present review concludes in favor of the role of multiparametric MRI in performing targeted biopsy of the prostate, in revealing Extra-Prostatic Extension (EPE) and in managing patients in active surveillance.

Secondarily, there is common agreement in the literature that the usage of multiparametric MRI may increase the number diagnosis of clinically significant prostate cancer and reduce the number of clinically insignificant ones, thus leading to a possible reduction in overdiagnosis and overtreatment.

Finally, multiparametric MRI is reported to be of help in planning the most appropriate surgical technique, thus leading to an overall improvement of the oncologic outcome and of the quality of life after radical surgery.

\section{Acknowledgments}

Funding: None.

\section{Footnote}

Provenance and Peer Review: This article was commissioned by the Guest Editor (Antonio Barile) for the series "Multimodality Advanced Imaging and Intervention in Gland Diseases" published in Gland Surgery. The article has undergone external peer review.

Conflicts of Interest: All authors have completed the ICMJE uniform disclosure form (available at http://dx.doi. org/10.21037/gs-20-561). The series "Multimodality Advanced Imaging and Intervention in Gland Diseases" was commissioned by the editorial office without any funding or sponsorship. The authors have no other conflicts of interest to declare.

Ethical Statement: The authors are accountable for all aspects of the work in ensuring that questions related to the accuracy or integrity of any part of the work are appropriately investigated and resolved.

Open Access Statement: This is an Open Access article distributed in accordance with the Creative Commons Attribution-NonCommercial-NoDerivs 4.0 International License (CC BY-NC-ND 4.0), which permits the noncommercial replication and distribution of the article with the strict proviso that no changes or edits are made and the original work is properly cited (including links to both the formal publication through the relevant DOI and the license). See: https://creativecommons.org/licenses/by-nc-nd/4.0/.

\section{References}

1. Zhang H, Shen Y, Pan J, et al. MRI features after prostatic artery embolization for the treatment of mediumand large-volume benign hyperplasia. Radiol Med 2018;123:727-34.

2. Beyhan M, Sade R, Koc E, et al. The evaluation of prostate lesions with IVIM DWI and MR perfusion parameters at 3T MRI. Radiol Med 2019;124:87-93.

3. Patel A, Jackson B. Low-dose radiation use in diagnostic imaging and cancer therapy settings. Radiol Med 2018;123:618-9.

4. Loi M, Incrocci L, Desideri I, et al. Prognostic impact of nodal relapse in definitive prostate-only irradiation. Radiol Med 2018;123:631-7.

5. Timon G, Jereczek-Fossa BA, Fersino S, et al. Nonpalliative radiotherapy in ab initio oligometastatic prostate cancer: an Italian national survey. Radiol Med 2019;124:211-7.

6. Borghetti P, Spiazzi L, Cozzaglio C, et al. Postoperative 
radiotherapy for prostate cancer: the sooner the better and potential to reduce toxicity even further. Radiol Med 2018;123:63-70.

7. Fersino S, Arcangeli S, Jereczek-Fossa BA, et al. GUROPA survey: genitourinary radiation oncology prescription attitudes. Radiol Med 2018;123:879-84.

8. Jereczek-Fossa BA, Surgo A, Maisonneuve P, et al. Late toxicity of image-guided hypofractionated radiotherapy for prostate: non-randomized comparison with conventional fractionation. Radiol Med 2019;124:65-78.

9. De Cicco L, Bracelli S. Fiducial markers implantation for prostate image-guided radiotherapy: a report on the transperineal approach. Radiol Med 2019;124:132-5.

10. Detti B, Baki M, Becherini C, et al. High-dose intensitymodulated radiation therapy as primary treatment of prostate cancer: genitourinary/gastrointestinal toxicity and outcomes, a single-institution experience. Radiol Med 2019;124:422-31.

11. Lehrich BM, Barnes L, Mesa A, et al. Response to "Is there a role for hydrogel spacer in post-prostatectomy radiotherapy setting?". Radiol Med 2019;124:1304-5.

12. Compagnone G, Padovani R, D'Avanzo MA, et al. Summary of the Italian inter-society recommendations for radiation protection optimization in interventional radiology. Radiol Med 2018;123:378-84.

13. Girometti R, Pancot M, Signor MA, et al. Multiparametric magnetic resonance imaging versus Partin tables and the Memorial Sloan-Kettering cancer center nomogram in risk stratification of patients with prostate cancer referred to external beam radiation therapy. Radiol Med 2018;123:778-87.

14. Pesapane F, Patella F, Fumarola EM, et al. The prostate cancer focal therapy. Gland Surg 2018;7:89-102.

15. Riccardi L, De Monte F, Cretti F. et al. Use of radiation dose index monitoring software in a multicenter environment for CT dose optimization. Radiol Med 2018;123:944-51.

16. Andriole GL, Crawford ED, Grubb RL 3rd, et al. Mortality results from a randomized prostate cancer screening trial. N Engl J Med 2009;360:1310-9.

17. Schröder FH, Hugosson J, Roobol MJ, et al. Screening and prostate cancer mortality in a randomized European study. N Engl J Med 2009;360:1320-8.

18. Malaspina S, De Giorgi U, Kemppainen J, et al. 68GaPSMA-PET: added value and future applications in comparison to the current use of choline-PET and mpMRI in the workup of prostate cancer. Radiol Med
2018;123:952-65.

19. Filograna L, Lenkowicz J, Cellini F, et al. Identification of the most significant magnetic resonance imaging (MRI) radiomic features in oncological patients with vertebral bone marrow metastatic disease: a feasibility study. Radiol Med 2019;124:50-7.

20. Petralia G, Padhani AR, Pricolo P, et al. Wholebody magnetic resonance imaging (WB-MRI) in oncology: recommendations and key uses. Radiol Med 2019;124:218-33.

21. Grassi R, Miele V, Giovagnoni A. Artificial intelligence: a challenge for third millennium radiologist. Radiol Med 2019;124:241-2.

22. Abdollahi H, Mofid B, Shiri I, et al. Machine learningbased radiomic models to predict intensity-modulated radiation therapy response, Gleason score and stage in prostate cancer. Radiol Med 2019;124: 555-67.

23. De Bari B, Mazzola R, Aiello D, et al. Could 68-Ga PSMA PET/CT become a new tool in the decision-making strategy of prostate cancer patients with biochemical recurrence of PSA after radical prostatectomy? A preliminary, monocentric series. Radiol Med 2018;123:719-25.

24. Petrillo M, Pesapane F, Fumarola EM, et al. State of the art of prostatic arterial embolization for benign prostatic hyperplasia. Gland Surg 2018;7:188-99.

25. Ierardi AM, Jannone ML, Brambillasca PM, et al. Bleeding after prostatectomy: endovascular management. Gland Surg 2019;8:108-14.

26. Cybulski AJ, Catania M, Brancato S, et al. Added value of MRI tractography of peri-prostatic nerve plexus to conventional T2-WI in detection of extra-capsular extension of prostatic cancer. Radiol Med 2019;124:946-54.

27. Basile A, Rebona A, Failla G, et al. Early post-procedural patients compliance and VAS after UAE through transradial versus transfemoral approach: preliminary results. Radiol Med 2018;123:885-9.

28. Giurazza F, Corvino F, Cavaglià E, et al. Arterial embolizations with microvascular plug in extracranial and intracranial districts: technical results. Radiol Med 2018;123:236-43.

29. Agostini A, Borgheresi A, Mari A, et al. Dual-energy CT: theoretical principles and clinical applications. Radiol Med 2019;124:1281-95.

30. Faiella E, Santucci D, Greco F, et al. Analysis of histological findings obtained combining US/mp-MRI fusion-guided biopsies with systematic US biopsies: mp- 
MRI role in prostate cancer detection and false negative. Radiol Med 2018;123:143-52.

31. Del Monte M, Leonardo C, Salvo V, et al. MRI/US fusion-guided biopsy: per-forming exclusively targeted biopsies for the early detection of prostate cancer. Radiol Med 2018;123:227-34.

32. Bloom JB, Gold SA, Hale GR, et al. "Super-active surveillance": MRI ultrasound fusion biopsy and ablation for less invasive management of prostate cancer. Gland Surg 2018;7:166-87.

33. Manetta R, Palumbo P, Gianneramo C, et al. Correlation between $\mathrm{ADC}$ values and Gleason score in evaluation of prostate cancer: multicentre experience and review of the literature. Gland Surg 2019;8:S216-22.

34. Epstein JI, Zelefsky MJ, Sjoberg DD, et al. A contemporary prostate cancer grading system: a validated alternative to the Gleason score. Eur Urol 2016;69:428-35.

35. Kane CJ, Eggener SE, Shindel AW, et al. Variability in Outcomes for Patients with Intermediate-risk Prostate Cancer (Gleason Score 7, International Society of Urological Pathology Gleason Group 2-3) and Implications for Risk Stratification: A Systematic Review. Eur Urol Focus 2017;3:487-97.

36. Valerio M, Donaldson I, Emberton M, et al. Detection of clinically significant prostate cancer using Magnetic Resonance Imaging-Ultrasound Fusion targeted biopsy: a systematic review. Eur Urol 2015;68:8-19.

37. Bratan F, Niaf E, Melodelima C, et al. Influence of imaging and histological factors on prostate cancer detection and localisation on multiparametric MRI: a prospective study. Eur Radiol 2013;23:2019-29.

38. Rouvière $\mathrm{O}$, Puech $\mathrm{P}$, Renard-Penna R, et al. Use of prostate systematic and targeted biopsy on the basis of multiparametric MRI in biopsy-naive patients (MRIFIRST): a prospective, multicentre, paired diagnostic study. Lancet Oncol 2019;20:100-109.

39. Drost FH, Osses DF, Nieboer D, et al. Prostate MRI, with or without MRI-targeted biopsy, and systematic biopsy for detecting prostate cancer. Cochrane Database Syst Rev 2019;4:CD012663.

40. Ziglioli F, Granelli G, Cavalieri D, et al. What chance do we have to decrease prostate cancer overdiagnosis and overtreatment? A narrative review. Acta biomed 2019;90:423-26.

41. Kasivisvanathan V, Rannikko AS, Borghi M, et al. MRItargeted or standard biopsy for prostate-cancer diagnosis.
N Engl J Med 2018;378:1767-77.

42. van der Leest $M$, Cornel E, Israël B, et al. Head-to-head comparison of transrectal ultrasound-guided prostate biopsy versus multiparametric prostate resonance imaging with subsequent magnetic resonance-guided biopsy in biopsy-naïve men with elevated prostate-specific antigen: a large prospective multicenter clinical study. Eur Urol 2019;75:570-8.

43. Budäus L, Graefen M, Salomon G, et al. The novel nomogram of Gleason sum upgrade: possible application for the eligible criteria of low dose rate brachytherapy. Int J Urol 2010;17:862-8.

44. Epstein JI, Feng Z, Trock BJ, et al. Upgrading and downgrading of prostate cancer from biopsy to radical prostatectomy: incidence and predictive factors using the modified Gleason grading system and factoring in tertiary grades. Eur Urol 2012;61:1019-24.

45. Itatani R, Namimoto T, Atsuji S, et al. Negative predictive value of multipara-metric MRI for prostate cancer detection: outcome of 5-year follow-up in men with negative findings on initial MRI studies. Eur J Radiol 2014;83:1740-5.

46. Arsov C, Becker N, Rabenalt R, et al. The use of targeted MR-guided prostate biopsy reduces the risk of Gleason upgrading on radical prostatectomy. J Cancer Res Clin Oncol 2015;141:2061-8.

47. Song W, Bang SH, Jeon HG, et al. Role of PI-RADS version 2 for prediction of upgrading in biopsy-proven prostate cancer with Gleason score 6. Clin Genitourin Cancer 2018;16:281-7.

48. Alqahtani S, Wei C, Zang Y, et al. Prediction of prostate cancer Gleason score upgrading from biopsy to radical prostatectomy using pre-biopsy multiparametric MRI PIRADS scoring system. Sci Rep 2020;10:7722.

49. D'Amico AV, Wittington R, Malcovicz SB, et al. The combination of preoperative Prostate Specific Antigen and postoperative pathological findings to predict Prostate Specific Antigen outcome in clinically localized prostate cancer. J Urol 1998;160:2096-101.

50. Maestroni U, Morandin F, Ferretti S, et al. Recurrence of prostate cancer after HIFU. Proposal of a novel predictive index. Acta Biomed 2018;89:220-6.

51. Jeong BC, Chalfin HJ, Lee SB, et al. The relationship between the extent of extraprostatic extension and survival following radical prostatectomy. Eur Urol 2015;67:342-6.

52. Mikel Hubanks J, Boorjian SA, Frank I, et al. The presence of extracapsular extension is associated with an 
increased risk of death from prostate cancer after radical prostatectomy for patients with seminal vesicle invasion and negative lymph nodes. Urol Oncol 2014;32:26.e1-7.

53. Feng TS, Sharif-Afshar AR, Wu J, et al. Multiparametric MRI improves accuracy of clinical nomograms for predicting extracapsular extension of prostate cancer of prostate cancer. Urology 2015;86:332-7.

54. Gupta RT, Faridi KF, Singh AA, et al. Comparing 3-T multiparametric MRI and the Partin tables to predict organ-confined prostate cancer after radical prostatectomy. Urol Oncol 2014;32:1292-9.

55. Morlacco A, Sharma V, Viers BR, et al. The incremental role of magnetic resonance imaging for prostate cancer staging before radical prostatectomy. Eur Urol 2017;71:701-4.

56. EAU - EANM - ESTRO - EUSR - SIOG Guidelines on Prostate Cancer 2020. Available online: https://uroweb. org/wp-content/uploads/EAU-EANM-ESTRO-ESURSIOG-Guidelines-on-Prostate-Cancer-2020v3.pdf

57. NCCN Guidelines on Prostate Cancer. Available online: https://nccn.org/professionals/physician_gls/pdf/prostate. pdf

58. Mehralivand S, Shih JH, Harmon S. et al. A grading system for the assessment of risk of extraporstatic extension of prostate cancer at multiparametric MRI. Radiology 2019;290:709-19.

59. Barentsz JO, Richenberg J, Clements R, et al. ESUR prostate MR Guidelines 2012. Eur Radiol 2012;22:746-57.

60. Costa DN, Passoni NM, Leyendecker JR, et al. Diagnostic utility of a Likert scale versus qualitative descriptors and length of capsular contact for determining extraprostatic tumor extension at multiparametric prostate MRI. AJR Am J Roentgenol 2018;210:1066-72.

61. Rosenkrantz AB, Shanbhogue AK, Wang A, et al. Length of capsular contact for diagnosing extraprostatic extension on prostate MRI: assessment of an optimal threshold. J Magn Reson Imaging 2016;43:990-7.

62. de Rooij M, Hamoen EHJ, Witjes JA, et al. Accuracy of magnetic resonance im-aging for focal staging of prostate cancer: a diagnostic meta-analysis. Eur Urol 2016;70:233-45.

63. Park JK, Kim M, Kim JK. Extraprostatic tumor extension: comparison of pre-operative multiparametric MRI criteria and histopathologic correlation after radical prostatectomy. Radiology 2020;5:192133.

64. Nyarangi-Dix J, Wiesenfarth M, Bonekamp D, et al. Combined Clinical Parameters and Multiparametric
Magnetic Resonance Imaging for the Prediction of Extraprostatic Disease-A Risk Model for Patient-tailored Risk Stratification When Planning Radical Prostatectomy. Eur Urol Focus 2020;6:1205-12.

65. Walz J, Epstein JI, Ganzer R, et al. A critical analysis of the current knowledge of surgical anatomy of the prostate related to optimisation of cancer control and preservation of continence and erection in candidates for radical prostatectomy: an update. Eur Urol 2016;70:301-11.

66. Schiavina R, Bianchi L, Borghesi M, et al. MRI displays the prostatic cancer anatomy and improve the bundles management before robot-assisted radical prostatectomy. J Endourol 2018;32:315-21.

67. Panebianco V, Salciccia S, Cattarino S, et al. Use of multiparametric MR with neurovascular bundle evaluation to optimize the oncological and functional management of patients considered for nerve-sparing radical prostatectomy. J Sex Med 2012;9:2157-66.

68. Hamdy FC, Donovan JL, Lane JA, et al. 10-Year Outcomes After Monitoring, Surgery, or Radiotherapy for Localized Prostate Cancer. N Engl J Med 2016;375:1415-24.

69. Thompson IM, Pauler DK, Goodman PJ, et al. Prevalence of prostate cancer among men with a Prostate-Specific Antigen level $<$ or $=4.0 \mathrm{ng}$ per milliliter. $\mathrm{N}$ Engl J Med 2004;350:2239-46.

70. Albertsen PC. Observational studies and the natural history of screen-detected prostate cancer. Curr Opin Urol 2015;25:232-7.

71. Bokhorst LP, Valdagni R, Rannikko A, et al. A decade of active surveillance in the PRIAS study: an update and evaluation of the criteria used to recommend a switch to active treatment. Eur Urol 2016;70:954-60.

72. San Francisco IF, Werner L, Regan MM, et al. Risk stratification and validation of prostate specific antigen density as independent predictor of progression in men with low risk prostate cancer during active surveillance. J Urol 2011;185:471-6.

73. Park BH, Jeon HG, Choo SH, et al. Role of multiparametric 3.0-Tesla magnetic resonance imaging in patients with prostate cancer eligible for active surveillance. BJU Int 2014;113:864-70.

74. Klotz L, Loblaw A, Sugar, L, et al. Active surveillance magnetic resonance imaging study (ASIST): results of a randomized multicenter prospective trial. Eur Urol 2019;75:300-9.

75. Siddiqui MM, Truong H, Rais-Bahrami S, et al. Clinical 
implications of a multiparametric magnetic resonance imaging based nomogram applied to prostate cancer active surveillance. J Urol 2015;193:1943-9.

Cite this article as: Ziglioli F, Maestroni U, Manna C, Negrini G, Granelli G, Greco V, Pagnini F, De Filippo M. Multiparametric MRI in the management of prostate cancer: an update—a narrative review. Gland Surg 2020;9(6):2321-2330. doi: $10.21037 / g s-20-561$
76. Schoots IG, Petrides N, Giganti F, et al. Magnetic Resonance Imaging in Active Surveillance of Prostate Cancer: A Systematic Review. Eur Urol 2015;67:627-36. 